\title{
Light pseudo-Goldstone Higgs boson from $S O(10)$ GUT with realistic phenomenology
}

\author{
Zurab Tavartkiladze* \\ Center for Elementary Particle Physics, ITP, Ilia State University, 0162 Tbilisi, Georgia
}

(Received 29 March 2018; published 9 July 2018)

\begin{abstract}
Within the supersymmetric $S O(10)$ grand unified theory (GUT), a new mechanism giving the light Higgs doublet as a pseudo-Goldstone mode is suggested. Realizing this mechanism, we present an explicit model with fully realistic phenomenology. In particular, desirable symmetry breaking and natural all-order hierarchy are achieved. The constructed model allows one to have a realistic fermion pattern, nucleon stability, and successful gauge coupling unification. The suggested mechanism opens prospects in the field for a novel $S O(10)$ GUT model building and for further investigations.
\end{abstract}

DOI: 10.1103/PhysRevD.98.015013

\section{INTRODUCTION}

Despite enormous success, the Standard Model (SM), failing to explain several observations, needs to be extended. Making steps beyond the SM, the grand unification theory (GUT) [1] is a highly motivated and elegant idea. The best candidate for GUT model building is the $S O(10)$ group [2], whose spinorial $16_{i}$-plets $(i=1$, 2 , 3), together with SM matter, include right-handed neutrino $\nu^{c}$ states (one per generation) $-16_{i}=$ $\left\{q, u^{c}, d^{c}, l, e^{c}, \nu^{c}\right\}_{i}$. Thus, within the SO(10) GUT, the most compelling seesaw neutrino mass generation mechanism [3] finds a natural realization, accommodating the data from various neutrino oscillation experiments. As shown, the same mass generation mechanism can generate the observed baryon asymmetry through leptogenesis [4]. Towards circumventing other problems, the supersymmetric (SUSY) extensions turn out to be a relief. SUSY ensures the stability of the weak scale. The SM's minimal SUSY extension (MSSM) leads to successful gauge coupling unification [5] (a necessary ingredient for GUTs) and includes the viable dark matter candidatethe neutralino.

Although there are numerous merits of the SUSY $S O(10)$ GUT (we study henceforth), the most challenging problem-the doublet-triplet (DT) splitting problem(together with other puzzles we address below) needs to be taken seriously [6]. The missing vacuum expectation value (VEV) mechanism, proposed earlier [7], enabling

\footnotetext{
"zurab.tavartkiladze@gmail.com
}

Published by the American Physical Society under the terms of the Creative Commons Attribution 4.0 International license. Further distribution of this work must maintain attribution to the author(s) and the published article's title, journal citation, and DOI. Funded by SCOAP ${ }^{3}$. one to have quite realistic constructions [8-13], triggered an intense investigation in the field [13]. An alternative, and perhaps most elegant, idea for resolving the DT problem is the pseudo-Goldstone boson (PGB) mechanism, which proposes the light Higgs as the PGB [14,15]. However, so far, attempts to realize this idea within GUTs, have been successful only within $S U(6)$ gauge symmetry [15-17]. Obviously, it would be very exciting to find a possibility for the PGB mechanism realization within the $S O(10)$ GUT.

In this paper, the first time within the SUSY SO(10) GUT, I propose the possibility for the Higgs doublet supermultiplets emerging as light PGBs. The model I present turns out to be fully realistic. Consistent GUT symmetry breaking is achieved, and the MSSM Higgs doublets are light pseudo-Goldstones, even after taking into account all allowed high-order operators. The model I construct offers a realistic fermion pattern, nucleon stability, and successful gauge coupling unification. Because of these issues (usually turning out to be severe problems for various GUTs), I find the presented $S O(10)$ model to be quite successful.

The mechanism proposed in this paper opens the door for future work to build varieties of PGB $S O(10)$ models and investigate their phenomenological implications.

\section{THE SETUP AND THE MECHANISM}

First, I describe the PGB mechanism within the SUSY $S O(10)$ GUT and then, on a concrete model, demonstrate its natural realization. The minimal superfield content, ensuring $\quad S O(10) \rightarrow S U(3)_{c} \times S U(2)_{L} \times U(1)_{Y} \equiv G_{\mathrm{SM}}$ breaking, is $\left\{45_{H}, 16_{H}, \overline{16}_{H}\right\}$ [and possibly some additional $S O(10)$ singlet states]. Thus, the symmetry-breaking scalar superpotential $W_{H}$ consists of three parts: 


$$
W_{H}=W_{H}^{(45)}+W_{H}^{(16)}+W_{H}^{(45,16)},
$$

where $W_{H}^{(45)}$ and $W_{H}^{(16)}$ involve separately $45_{H}$ and $\left\{16_{H}, \overline{16}_{H}\right\}$-plets, respectively. The $W_{H}^{(45)}$, including some powers of $45_{H}$ (such as $45_{H}^{2}, 45_{H}^{4}, \ldots$ ), is responsible for fixing the VEV $\left\langle 45_{H}\right\rangle$ towards the desirable direction. The $W_{H}^{(16)}$ fixes the VEVs of $16_{H}$ and $\overline{16}_{H}$ in their $\nu_{H}^{c}, \bar{\nu}_{H}^{c}$ scalar components and can be $W_{H}^{(16)}=S\left(i \overline{16}_{H} 16_{H}+v_{R}^{2}\right)$, where $S$ is the $S O(10)$ singlet. The condition $F_{S}=0$, together with $D$-flatness, would fix $\left\langle\nu_{H}^{c}\right\rangle=\left\langle\bar{\nu}_{H}^{c}\right\rangle=v_{R}$. For us, it is essential that the terms like $\left(\overline{16}_{H} 16_{H}\right)^{2}$ and $16_{H} 16_{H} 45_{H}^{2 n+1}$ are absent or are properly suppressed (will be explained below). The superpotential $W_{H}^{(45,16)}$ includes couplings of $\overline{16}_{H} 16_{H}$ with only even powers of $45_{H}$ (this can be guaranteed by discrete symmetries), such as $\overline{16}_{H} 45_{H}^{2} 16_{H}$. Without $W_{H}^{(45,16)}$, the $W_{H}$ has global symmetry $S O(10)_{45} \times$ $U(16)$ [e.g., $W_{H}^{(45)}$ possesses $S O(10)_{45}$ symmetry, while the $W_{H}^{(16)}$ has $U(16)$ global symmetry]. With the $\operatorname{VEVs}\left\langle\nu_{H}^{c}\right\rangle$ and $\left\langle\bar{\nu}_{H}^{c}\right\rangle$, the breaking $U(16) \rightarrow U(15)$ happens and pseudo-Goldstone states emerge. Clearly, those modes (at least some of those) would gain masses by including the terms of $W_{H}^{(45,16)}$. Our goal is to see if it is possible to arrange the couplings such that MSSM Higgs doublets emerge as light pseudo-Goldstones while all remaining states acquire large masses. It turns out that in this setup this is indeed possible.

Assume that the $45_{H}$ has a VEV towards the $B-L$ direction $\left\langle 45_{H}\right\rangle \sim V_{B-L}$. Thus, with $S O(10) \rightarrow S U(4)_{c} \times$ $S U(2)_{L} \times S U(2)_{R}$ decomposition

$45_{H}=\Sigma(15,1,1)+\Sigma_{R}(1,1,3)+\Sigma_{L}(1,3,1)+(6,2,2)$,

the $S U(4)_{c}$ 's adjoint $\Sigma(15,1,1)$ have the VEV $\langle\Sigma\rangle=$ $V_{B-L} \cdot \operatorname{Diag}(1,1,1,-3)$, causing the breaking of $S O(10)$ gauge symmetry down to $G_{3221} \equiv S U(3)_{c} \times S U(2)_{L} \times$ $S U(2)_{R} \times U(1)_{B-L}$. With this breaking, the 30 states (from $45_{H}$ ) absorbed by superheavy gauge fields become genuine Goldstones. These are the fragment $(6,2,2)$ [given in (2)] plus $S U(3)_{c}$ 's triplet-antitriplet pair $3_{\Sigma}, \overline{3}_{\Sigma}$ coming from $\Sigma(15,1,1)$. The remaining fragments of $45_{H}$ should gain large masses. Moreover, with $W_{H}^{(16)}$ 's terms, such as $\lambda_{S} S\left(i \overline{16}_{H} 16_{H}+v_{R}^{2}\right)$, one can have $\left\langle\nu_{H}^{C}\right\rangle=\left\langle\bar{\nu}_{H}^{C}\right\rangle=v_{R}$, inducing the breaking $G_{3221} \rightarrow G_{\mathrm{SM}}$. The spontaneous breaking $W_{H}^{(16)}$,s global $U(16)$ would give unwanted pseudo-Goldstone modes. However, the superpotential $W_{H}^{(45,16)} \supset \frac{\lambda i}{4 ! M_{*}} \overline{16}_{H} 45_{H}^{2} 16_{H}$ coupling will break $U(16)$ but, due to the $\left\langle 45_{H}\right\rangle$ configuration, render some global symmetries. In particular, using in this coupling the VEV $\left\langle 45_{H}\right\rangle=\langle\Sigma\rangle \propto Q_{B-L}$ [see Eq. (2) and the comment below it], the bilinear terms with respect to the states $16_{H}=\left\{f_{H}\right\}, \overline{16}_{H}=\left\{\bar{f}_{H}\right\}$, where $f_{H}=\left\{\nu_{H}^{c}, e_{H}^{c}\right.$, $\left.l_{H}, u_{H}^{c}, d_{H}^{c}, q_{H}\right\}$, will be

$$
\begin{aligned}
& \frac{\lambda}{M_{*}} \overline{16}_{H} 45_{H}^{2} 16_{H} \rightarrow \lambda \frac{V_{B-L}^{2}}{M_{*}} \sum_{f} \bar{f}_{H}\left(Q_{B-L}^{f}\right)^{2} f_{H}+\cdots \\
& =\lambda \frac{V_{B-L}^{2}}{M_{*}}\left(\bar{\nu}_{H}^{c} \nu_{H}^{c}+\bar{e}_{H}^{c} e_{H}^{c}+\bar{l}_{H} l_{H}\right) \\
& \quad+\lambda \frac{V_{B-L}^{2}}{M_{*}} \frac{1}{9}\left(\bar{u}_{H}^{c} u_{H}^{c}+\bar{d}_{H}^{c} d_{H}^{c}+\bar{q}_{H} q_{H}\right)+\cdots
\end{aligned}
$$

where I have displayed terms not respecting the $U(16)$ global symmetry. However, due to the relation $M_{f_{H} \bar{f}_{H}} \propto\left(Q_{B-L}^{f}\right)^{2}$, as seen from (3), the second and last lines possess $U(4)$ and $U(12)$ symmetries, respectively. The $U(4)$ global symmetry, which has leptonic states, is spontaneously broken down to $U(3)$ by the VEVs $\left\langle\nu_{H}^{C}\right\rangle=\left\langle\bar{\nu}_{H}^{C}\right\rangle=v_{R}$. Because of $U(4) \rightarrow$ $U(3)$ breaking, the seven Goldstones emerge. Among them, three-one SM singlet and the pair $e_{H}^{c}, \bar{e}_{H}^{c}$-are genuine Goldstones absorbed by the coset $\left[S U(2)_{R} \times\right.$ $\left.U(1)_{B-L}\right] / U(1)_{Y}$ gauge fields. The states $l_{H}$ and $\bar{l}_{H}$, having the quantum numbers of the MSSM Higgs superfields, emerge as light (as desired) pseudo-Goldstone modes. The remaining fragments, given in the last line in (3), will be heavy.

However, for this mechanism to be realized, some care needs to be exercised. The $\lambda$ coupling term, with nonzero $\left\langle\nu_{H}^{C}\right\rangle=\left\langle\bar{\nu}_{H}^{C}\right\rangle=v_{R}$, would also trigger the VEV of $45_{H}$ in the $I_{3 R}$ direction, i.e., the VEV of $\Sigma_{R}(1,1,3)$ of (2) with the $\left\langle\Sigma_{R}\right\rangle=V_{R} \cdot \operatorname{Diag}(1,-1)$ configuration in $S U(2)_{R}$ space. As expected, $V_{R} \sim \lambda v_{R}^{2} / V_{B-L}$. This, in (3), would introduce $U(4)$ symmetry-breaking mass terms $\sim \lambda^{2} v_{R}^{2} / M_{*}$. Thus, the PGB's mass-the $\mu$ term-will be $\mu \sim \lambda^{2} v_{R}^{2} / M_{*}$. For its proper suppression, for $v_{R}=10^{16-17} \mathrm{GeV}, M_{*} \simeq$ $2.4 \times 10^{18} \mathrm{GeV}$, we need $\lambda \lesssim 10^{-6}$. Besides this, terms such as $\overline{16}_{H} 45_{H}^{2 n+1} 16_{H}$ and $\left(16_{H} \overline{16}_{H}\right)^{k+2} \quad(n, k=0,1,2, \ldots)$ should be absent or adequately suppressed. Below, I present the model where all this is naturally realized; i.e., smallness or absence of any coupling will follow from the symmetries (we will invoke).

\section{MODEL WITH ALL-ORDER NATURAL HIERARCHY}

Realizing the mechanism described above, we augment the SUSY $S O(10)$ GUT by $\mathcal{U}(1)_{A} \times \mathcal{Z}_{4}$ symmetry, where $\mathcal{U}(1)_{A}$ is an anomalous gauge symmetry and $\mathcal{Z}_{4}$ is discrete $R$ symmetry. For symmetry breaking and all-order DT hierarchy, the anomalous $\mathcal{U}(1)_{A}$ was first applied in Ref. [17] [within the PGB $S U(6)$ GUT] and was proven to be very efficient for realistic $S O(10)$ model building $[9,12]$. Besides the states $45_{H}, 16_{H}$, and $\overline{16}_{H}$, we introduce two $S O(10)$ singlet superfields $S$ and $S_{1}$, which will be used in superpotential $W_{H}$ of Eq. (1). In Table I, I display the $\mathcal{U}(1)_{A} \times \mathcal{Z}_{4}$ transformation properties of these fields together with other states (introduced and discussed later on). Note that, under $\mathcal{Z}_{4}$ symmetry, the whole 
TABLE I. $\mathcal{U}(1)_{A}$ and $\mathcal{Z}_{4}$ charges $Q_{i}$ and $\omega_{i}$ of the superpotential and superfield $\phi_{i}$. The transformations under $\mathcal{U}(1)_{A}$ and $\mathcal{Z}_{4}$ are, respectively, $\phi_{i} \rightarrow e^{i Q_{i}} \phi_{i}$ and $\phi_{i} \rightarrow e^{i \omega_{i}} \phi_{i} ; \omega=\frac{2 \pi}{4}$.

\begin{tabular}{cccccccccc}
\hline \hline & $W$ & $45_{H}$ & $16_{H}$ & $\overline{16}_{H}$ & $S$ & $S_{1}$ & $10_{H}$ & $10^{\prime}{ }_{H}$ & $16_{i}$ \\
\hline$Q_{i}$ & 0 & 0 & -5 & 1 & 0 & 4 & -6 & 6 & 3 \\
$\omega_{i}$ & $2 \omega$ & $\omega$ & $-\omega$ & $\omega$ & $2 \omega$ & 0 & 0 & $\omega$ & $\omega$ \\
\hline \hline
\end{tabular}

superpotential transforms as $W \rightarrow-W$. Thus, the relevant superpotential couplings are

$$
\begin{aligned}
W_{H}^{(45)}= & \frac{1}{2} M_{45} \operatorname{tr}\left(45_{H}^{2}\right)+\frac{\lambda_{1}}{M_{*}^{3}}\left[\operatorname{tr}\left(45_{H}^{2}\right)\right]^{3} \\
& +\frac{\lambda_{2}}{M_{*}^{3}} \operatorname{tr}\left(45_{H}^{2}\right) \operatorname{tr}\left(45_{H}^{4}\right)+\frac{\lambda_{3}}{M_{*}^{3}} \operatorname{tr}\left(45_{H}^{6}\right), \\
W_{H}^{(16)}= & -\lambda_{S} S\left(i \frac{S_{1}}{M_{*}} \overline{16}_{H} 16_{H}+\Lambda^{2}\right), \\
W_{H}^{(45,16)}= & \frac{\bar{\lambda} i S_{1}}{4 ! M_{*}^{2}} \overline{16}_{H} 45_{H}^{2} 16_{H} .
\end{aligned}
$$

With anomalous $\mathcal{U}(1)_{A}$, having the string origin, the Fayet-Iliopoulos term $\int d^{4} \theta \xi V_{A}$ is always generated [18], and the corresponding $D$-term potential is

$V_{D}=\frac{g_{A}^{2}}{8}\left(\xi+\sum_{i} Q_{i}\left|\phi_{i}\right|^{2}\right)^{2} \rightarrow \frac{g_{A}^{2}}{8}\left(\xi-4 v_{R}^{2}+4\left|\left\langle S_{1}\right\rangle\right|^{2}\right)^{2}$.

At the last stage in (5), we have taken into account that $\left\langle\nu_{H}^{c}\right\rangle=\left\langle\bar{\nu}_{H}^{c}\right\rangle \equiv v_{R}$, which ensures the vanishing of all $D$ terms of $S O(10)$. With $\xi>0$ and looking for the solution of $\left\langle S_{1}\right\rangle \ll v_{R}$, the condition $\left\langle D_{A}\right\rangle=0$ from (5) gives

$$
v_{R} \simeq 0.5 \sqrt{\xi}
$$

Next, we investigate the symmetry-breaking pattern from the superpotential couplings given in (4). With the branching $\overline{16} \times 16=1+45+210$ and noting that $45_{H}^{2}$ identically vanishes in the antisymmetric 45 channel, the remaining relevant contraction [breaking global $U(16)$ symmetry] is in the 210 channel. Thus, under the $\overline{16}_{H} 45_{H}^{2} 16_{H}$ term, we assume the invariant $\left(\overline{16}_{H} 16_{H}\right)_{210}$. $\left(45_{H}^{2}\right)_{210}$ (see the Appendix for more details).

Writing $45_{H}$ 's VEV in $S O(10)$ group space as

$$
\left\langle 45_{H}\right\rangle=i \sigma_{2} \otimes \operatorname{Diag}\left(V_{B-L}, V_{B-L}, V_{B-L}, V_{R}, V_{R}\right)
$$

and imposing $F_{\phi_{i}}=0$, from the superpotential couplings (4), taking into account that $v_{R}$ is fixed as (6), we obtain

$$
\begin{gathered}
\left\langle S_{1}\right\rangle \simeq \frac{\Lambda^{2}}{v_{R}^{2}} M_{*}, \\
\langle S\rangle=-\frac{3 \bar{\lambda}}{\lambda_{S} M^{*}} V_{B-L}^{2}\left(1+\frac{2 V_{R}}{V_{B-L}}+\frac{V_{R}^{2}}{3 V_{B-L}^{2}}\right), \\
6\left(36 \lambda_{1}+6 \lambda_{2}+\lambda_{3}\right) V_{B-L}^{4}+16\left(18 \lambda_{1}+\lambda_{2}\right) V_{B-L}^{2} V_{R}^{2} \\
+8\left(12 \lambda_{1}+\lambda_{2}\right) V_{R}^{4} \\
=-M_{45} M_{*}^{3}+\bar{\lambda}\left\langle S_{1}\right\rangle v_{R}^{2} M_{*}\left(1+\frac{V_{R}}{V_{B-L}}\right), \\
\left(16 \lambda_{1}+4 \lambda_{2}+\lambda_{3}\right) V_{R}^{5}+4\left(12 \lambda_{1}+\lambda_{2}\right) V_{B-L}^{2} V_{R}^{3} \\
+2\left(18 \lambda_{1}+\lambda_{2}\right) V_{B-L}^{4} V_{R}+\frac{1}{6} M_{45} M_{*}^{3} V_{R} \\
=\frac{1}{4} \bar{\lambda}\left\langle S_{1}\right\rangle v_{R}^{2} V_{B-L} M_{*}\left(1+\frac{V_{R}}{3 V_{B-L}}\right) .
\end{gathered}
$$

From (8)-(10), one can see that the desirable VEV configuration is obtained. Indeed, from (8), with $v_{R} \sim$ $10^{17} \mathrm{GeV}$ [fixed from (6)] and $\Lambda=\left(10^{13}-10^{14}\right) \mathrm{GeV}$, we obtain $\frac{\left\langle S_{1}\right\rangle}{M_{*}} \simeq 10^{-8}-10^{-6}$. Thus, an effective, and suppressed, $\lambda$ coupling (ensuring suppressed Higgs mass) is generated. Small $\left\langle S_{1}\right\rangle$ also ensures naturally suppressed $V_{R}$ (in a limit $\left\langle S_{1}\right\rangle \rightarrow 0$, one has the solution $V_{R} \rightarrow 0$ ). At the leading order, in powers of $\frac{\left\langle S_{1}\right\rangle}{M_{*}}$, from (8)-(10) we get

$$
\text { with } \begin{aligned}
\frac{\Lambda}{v_{R}} \ll 1: V_{B-L} & \simeq\left(\frac{-M_{45} M_{*}^{3}}{6\left(36 \lambda_{1}+6 \lambda_{2}+\lambda_{3}\right)}\right)^{1 / 4}, \\
\frac{\left\langle S_{1}\right\rangle}{M_{*}} & \simeq \frac{\Lambda^{2}}{v_{R}^{2}}, \\
V_{R} & \simeq-\bar{\lambda} \frac{\left\langle S_{1}\right\rangle}{M_{*}} \frac{v_{R}^{2} M_{*}^{2}}{4\left(4 \lambda_{2}+\lambda_{3}\right) V_{B-L}^{3}}, \\
\langle S\rangle & \simeq-\frac{3 \bar{\lambda}}{\lambda_{S}} \frac{V_{B-L}^{2}}{M_{*}} .
\end{aligned}
$$

For the masses of $l_{H}$ and $\bar{l}_{H}$, coming from the $16_{H}$ and $\overline{16}_{H}$, we have (in the Appendix, I present the decomposition helping to compute the masses of this and remaining fragments)

$$
\begin{aligned}
M_{\bar{l}_{H} l_{H}} & =\frac{-6 \bar{\lambda}\left\langle S_{1}\right\rangle V_{B-L} V_{R}}{M_{*}^{2}}\left(1-\frac{V_{R}}{3 V_{B-L}}\right) \\
& \simeq\left(\frac{\Lambda}{v_{R}}\right)^{4} \frac{3 \bar{\lambda}^{2} v_{R}^{2} M_{*}}{2\left(4 \lambda_{2}+\lambda_{3}\right) V_{B-L}^{2}}
\end{aligned}
$$

The latter's value, i.e., the $\mu$ term, with $v_{R} \sim V_{B-L}$ and for $\frac{\Lambda}{v_{R}} \sim(1-2) \times 10^{-4}$ will be $\sim 1-10 \mathrm{TeV}$ - of desirable magnitude. Allowed higher-order operator $\frac{1}{M_{*}^{5}}\left(S_{1} \overline{16}_{H} 45_{H} 16_{H}\right)^{2}$ is harmless, because it contributes to the $\mu$ term by the 
amount $\sim \frac{\left\langle S_{1}\right\rangle^{2} V_{B-L}^{2} v_{R}^{2}}{M_{*}^{5}} \lesssim 10^{-2} \mathrm{GeV}$ (for $V_{B-L}, v_{R} \sim M_{*} / 30$ ).

The masses of colored modes (from $16_{H}, \overline{16}_{H}$ ) are

$$
M_{\bar{q}_{H} q_{H}} \simeq M_{\bar{u}_{H}^{c} u_{H}^{c}} \simeq M_{\bar{d}_{H}^{c} d_{H}^{c}} \equiv M_{T_{1}} \simeq-4 \bar{\lambda}\left(\frac{\Lambda}{v_{R}}\right)^{2} \frac{V_{B-L}^{2}}{M_{*}},
$$

around $\sim 10^{8} \mathrm{GeV}$.

Below, I show how MSSM Higgs doublets can have desirable couplings to the MSSM matter and how nucleon stability is ensured [19]. Before discussing these, I give the scalar sector's extension, which ensures desirable Yukawa couplings and realistic phenomenology. I introduce two scalar superfields in the fundamental representation (10-plets) of $S O(10): 10_{H}$ and $10_{H}^{\prime}$ with $\mathcal{U}(1)_{A} \times \mathcal{Z}_{4}$ charges given in Table I. Thus, the relevant superpotential couplings are

$$
\begin{aligned}
W_{H}^{(16,10)}= & \alpha_{1} \frac{S_{1}}{M_{*}} \overline{16}_{H} \overline{16}_{H} 10_{H}+\lambda^{\prime} 10_{H} 45_{H} 10^{\prime}{ }_{H} \\
& +\alpha_{2} \frac{\left(\overline{16}_{H} 16_{H}\right)^{3}}{M_{*}^{5}} 10_{H}^{\prime}{ }_{H} 10^{\prime}{ }_{H}
\end{aligned}
$$

( $\alpha_{1,2}$ and $\lambda^{\prime}$ are dimensionless couplings), which, with notations $\left\{l_{H}, \bar{l}_{H}\right\} \equiv\left\{h_{d}^{16_{H}}, h_{u}^{\overline{16}_{H}}\right\}$, give the doublet mass matrix:

$$
\begin{aligned}
& h_{d}^{16_{H}} \quad h_{d}^{10_{H}} \quad h_{d}^{10^{\prime}{ }_{H}} \\
& M_{D}=\begin{array}{l}
h_{u}^{\overline{16}_{H}} \\
h_{u}^{10_{H}} \\
h_{u}^{10^{\prime}{ }_{H}}
\end{array}\left(\begin{array}{ccc}
0 & \bar{M} & 0 \\
0 & 0 & \lambda^{\prime} V_{R} \\
0 & -\lambda^{\prime} V_{R} & M^{\prime}
\end{array}\right)+\mathcal{O}(\mathrm{TeV}), \\
& \text { with } \quad \bar{M}=\alpha_{1} \frac{\left\langle S_{1}\right\rangle v_{R}}{M_{*}}, \quad M^{\prime}=\alpha_{2} \frac{v_{R}^{6}}{M_{*}^{5}} \text {. }
\end{aligned}
$$

In (15), the $\mathcal{O}(\mathrm{TeV})$ stand for possible corrections of the order of (1-10) $\mathrm{TeV}$ (I will comment shortly) and are harmless.

From (15), we see that one doublet pair, identified with MSSM Higgs superfields $h_{u}$ and $h_{d}$, is light. The remaining doublets (denoted as $D_{1,2}$ and $\bar{D}_{1,2}$ ) are heavy. Analyzing the matrix (15), the distribution of $h_{u}$ and $h_{d}$ in original states can be found:

$h_{u}^{10_{H}}=e^{i \phi_{x}}|x| h_{u}+\cdots, \quad h_{d}^{16_{H}}=h_{d}$,
with $\frac{1}{|x|}=\frac{1}{\left|M^{\prime}\right|}\left(\left|M^{\prime}\right|^{2}+\left|\lambda^{\prime} V_{R}\right|^{2}+\frac{\left|\lambda^{\prime} V_{R}\right|^{4}}{|\bar{M}|^{2}}\right)^{1 / 2}$,

the composition crucial for building a realistic fermion pattern (especially for large top Yukawa coupling $\lambda_{t}$ ).
As far as other operators, allowed by symmetries, are concerned, the couplings $\frac{S S_{4} 45_{H}}{M_{*}^{3}} 16_{H} 16_{H} 10_{H}^{\prime}$ and $\frac{S 45_{H}}{M_{*}^{6}}\left(\overline{16}_{H} 16_{H}\right)^{2} \overline{16}_{H} \overline{16}_{H} 10^{\prime}{ }_{H}$ with $\frac{\langle S\rangle}{M_{*}} \sim 10^{-4}, \frac{\left\langle S_{1}\right\rangle}{M_{*}}=\frac{\Lambda^{2}}{v_{R}^{2}} \sim 10^{-8}$, and $v_{R}, V_{B-L} \sim \frac{M_{*}}{30}$ will contribute to the $\mu$ term by an amount of $\sim$ few $\mathrm{TeV}$ and therefore are harmless [20].

\section{YUKAWA COUPLINGS AND PROTON STABILITY}

All MSSM matter is embedded in $S O(10)$ 's $16_{i}$-plets $(i=1,2,3)$. Their $\mathcal{U}(1)_{A} \times \mathcal{Z}_{4}$ charges are given in Table I. The effective operators, generating up- and down-type quark and charged lepton masses, are

$W_{Y}=Y_{U}^{i j} 16_{i} 16_{j} 10_{H}+\frac{45_{H}^{2}}{\left\langle\overline{16}_{H} 16_{H}\right\rangle} \frac{Y^{i j}}{M_{*}} 16_{i} 16_{j} 16_{H} 16_{H}$.

The first term is responsible for the up-type quark Yukawa couplings. According to (17), the $10_{H}$-plet includes the $h_{u}$ by the weight $|x|$, which with $\left|\lambda^{\prime} V_{R}\right| \sim|\bar{M}| \sim\left|M^{\prime}\right|$ can naturally be $|x| \approx 0.5-1$. Therefore, the $\lambda_{t}$ coupling can naturally have a desirable value. On the other hand, the $h_{d}$ entirely resides in the $16_{H}$-plet, and down quark and charged lepton masses are emerging from the second term of (18), which can be obtained by integrating out heavy vectorlike fermion superfields. For instance, with additional $10_{f}$ states [with $\mathcal{U}(1)_{A} \times \mathcal{Z}_{4}$ charges $Q_{10_{f}}=2, \omega_{10_{f}}=\omega$ ] and couplings $\left[\frac{y^{i \alpha}}{M_{*}} 16_{H} 45_{H} 16_{i} 10_{f \alpha}-\frac{\kappa^{\alpha \beta}}{4 M_{*}} \overline{16}_{H} 16_{H} 10_{f \alpha} 10_{f \beta}\right]$, one can see that integration of $10_{f}$ states (gaining masses after substitution of the VEV $\left.\left\langle\overline{16}_{H} 16_{H}\right\rangle\right)$ leads to the second term of (18) with $Y^{i j} \simeq\left(y \kappa^{-1} y^{T}\right)^{i j}$. Note that the $45_{H}^{2}$ can couple to the third family by the contraction $\operatorname{tr}\left(45_{H}^{2}\right)$ [i.e., $\frac{\operatorname{tr}\left(45_{H}^{2}\right)}{\left\langle 16_{H} 16_{H}\right\rangle} 16_{3} 16_{3} 16_{H} 16_{H}$ ] and, therefore, one can have $\lambda_{b}=\lambda_{\tau}$-the bottom-tau unification-at the GUT scale. The couplings $y^{i \alpha}$ and $\kappa^{\alpha \beta}$ can also be obtained via renormalizable interactions. For example, having additional $16_{f}$ and $\overline{16}_{f}$ states [with $Q_{16_{f}}=-Q_{\overline{16_{f}}}=3$ and $\omega_{16_{f}}=2 \omega, \omega_{\overline{16}_{f}}=0$ ] and superpotential terms $16_{i} 45_{H} \overline{16}_{f}+16_{H} 10_{f} 16_{f}+M_{f} \overline{16}_{f} 16_{f}$, decoupling of $16_{f}$ and $\overline{16}_{f}$ generates a term with $y^{i \alpha}$ coupling above. I will not discuss here more details (should be pursued elsewhere) but emphasize that after the GUT symmetry breaking some fragments from $10_{f}, 16_{f}$, and $\overline{16}_{f}$ can have masses below the GUT scale and their contribution, as thresholds, may play an important role for the gauge coupling unification (discussed at the end).

The right-handed neutrinos $\nu_{i}^{c}$ are gaining masses $\left(\equiv M_{R}\right)$ via the effective operator $\left(16_{i} \overline{16}_{H}\right)^{2} \frac{\left(\overline{16}_{H} 16_{H}\right)^{2}}{\langle S\rangle M_{*}^{4}}$, which can be generated by decoupling the $S O(10)$ singlets with masses $\sim\langle S\rangle$. With $\frac{\langle S\rangle}{M_{*}} \sim 10^{-4}, M_{R} \sim \frac{v_{R}^{6}}{\langle S\rangle M_{*}^{4}} \approx 10^{14} \mathrm{GeV}$ indeed of desirable values, generating the light neutrino masses $\sim 0.1 \mathrm{eV}$ via the seesaw mechanism [3]. Before 
discussing $d=5$ proton decay, note that $10_{H}^{\prime}$ 's couplings with matter $\frac{1}{M_{*}^{8}}\left(\overline{16}_{H} 16_{H}\right)^{3} S 45_{H} 16_{i} 16_{j} 10_{H}^{\prime} \rightarrow$ $\sim 10^{-14} 16_{i} 16_{j} 10_{H}^{\prime}$ are so suppressed that they should be completely ignored.

The couplings in (18), after substituting appropriate VEVs, besides the Yukawa interactions

$W_{Y} \rightarrow\left(q Y_{U} u^{c}+l Y_{U} \nu^{c}\right) h_{u}+\left(q Y_{D} d^{c}+e^{c} Y_{E} l\right) h_{d}$,

also generate couplings

$$
\frac{1}{2} q Y_{U} q T_{10_{H}}+q Y_{U} l \bar{T}_{10_{H}}+q Y_{q l} l \bar{T}_{16_{H}}
$$

(with notation $\bar{T}_{16_{H}}=d_{H}^{c}$ ). Integration of the triplet states, forming the mass matrix coupling $T^{T} M_{T} \bar{T}$, leads to a baryon number-violating $L L L L d=5$ operator:

$$
\mathcal{O}_{d=5}^{L L L L}=\frac{1}{2}\left[q Y_{q l} l\left(M_{T}^{-1}\right)_{12}+q Y_{U} l\left(M_{T}^{-1}\right)_{22}\right] q Y_{U} q .
$$

The triplet mass matrix, derived from the couplings (14) and taking into account (13), has the form

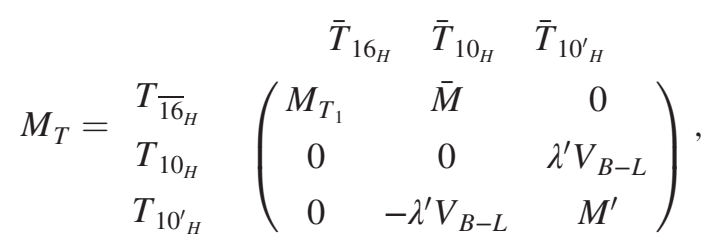

from which $\left(M_{T}^{-1}\right)_{12}=\frac{-\bar{M} M^{\prime}}{M_{T_{1}}\left(\lambda^{\prime} V_{B-L}\right)^{2}}$ and $\left(M_{T}^{-1}\right)_{22}=\frac{M^{\prime}}{\left(\lambda^{\prime} V_{B-L}\right)^{2}}$. For $\lambda^{\prime} \simeq 1$, the mass scales $M_{T_{1}} \simeq 10^{8} \mathrm{GeV}$, $\bar{M} \simeq 3 \times 10^{11} \mathrm{GeV}$, and $M^{\prime} \simeq 8 \times 10^{11} \mathrm{GeV}$, whose values are dictated from (13), (16), and desirable phenomenology [remember, the value $V_{R} \approx 5 \times 10^{11} \mathrm{GeV}$ obtained from (11) and the need for $|x| \approx 0.5-1$ of (17) giving the needed value of $\lambda_{t}$, so we take $\left.\left|\lambda^{\prime} V_{R}\right|=5 \times 10^{11} \mathrm{GeV}\right]$, and with $V_{B-L} \simeq 8 \times 10^{16} \mathrm{GeV}$, we obtain $\left(M_{T}^{-1}\right)_{12} \simeq \frac{1}{2.7 \times 10^{18} \mathrm{GeV}}$ and $\left(M_{T}^{-1}\right)_{22} \simeq \frac{1}{8 \times 10^{21} \mathrm{GeV}}$. These ensure the adequate suppression of $d=5$ operators in (21) [21]. Remarkably, although the color triplets from $16_{H}$ and $\overline{16}_{H}$ have intermediate mass $\left[\sim 10^{8} \mathrm{GeV}\right.$; see Eq. (13)], the model allows one to have a stable nucleon and therefore be fully self-consistent.

\section{GAUGE COUPLING UNIFICATION}

Finally, let us discuss the impact of some intermediate states, populating the "desert" between the electroweak and GUT scales. From (22), one triplet pair has mass $M_{T_{1}}$ given in (13) (together with the remaining colored states). We denote the masses of the remaining two triplet-antitriplet pairs by $M_{T_{2,3}}$. These and heavy doublets' masses $M_{D_{1,2}}$ are found from (22) and (15): $M_{T_{2,3}} \simeq\left|\lambda^{\prime} V_{B-L}\right|$ and $2 M_{D_{1,2}}^{2}=$ $|\bar{M}|^{2}+\left|M^{\prime}\right|^{2}+2\left|\lambda^{\prime} V_{R}\right|^{2} \pm\left[\left(|\bar{M}|^{2}-\left|M^{\prime}\right|^{2}\right)^{2}+4\left|\lambda^{\prime} V_{R} M^{\prime}\right|^{2}\right]^{1 / 2}$. Moreover, from $45_{H}$ come a colored octet (with mass $M_{8}$ ) and $S U(2)_{L}$ triplet (with mass $M_{3}$ ). One physical $e^{c}+\bar{e}^{c}$ pair, with mass $M_{e_{H}^{c}}$, comes from the superposition of the corresponding fragments from $45_{H}$ and $16_{H}, \overline{16}_{H}$ (the orthogonal combination is an absorbed Goldstone). A similar thing happens with the scalar supermultiplets having the quantum numbers of $u_{H}^{c}, q_{H}, \bar{u}_{H}^{c}$, and $\bar{q}_{H}$. We will denote the masses of the corresponding physical states by $M_{u_{S}^{c}}$ and $M_{q_{S}}$. From Eqs. (4), (11), and (13), we have $\frac{M_{8}}{8 \lambda_{2}+4 \lambda_{3}} \simeq \frac{M_{3\left(e_{H}^{c}\right)}}{4 \lambda_{2}+\lambda_{3}} \simeq 6 \frac{V_{B-L}^{4}}{M_{*}^{3}}$ and $M_{u_{S}^{c}\left(q_{S}\right)} \simeq M_{\bar{d}_{H}^{c} d_{H}^{c}} \simeq M_{T_{1}}$. From the vectorlike heavy matter $16_{f}, \overline{16}_{f}, 10_{f}$ [discussed after Eq. (18)], we assume that $n_{f}$ pairs of $u_{f}^{c}+\bar{u}_{f}^{c}$ and $l_{f}+\bar{l}_{f}$ happen to have masses $M_{u_{f}^{c}}$ and $M_{l_{f}}$, respectively, below the GUT scale $M_{G}$. Thus, their thresholds will also contribute to the gauge coupling running. With all this, imposing the unification condition for the gauge couplings at scale $M_{G}$, from renormalisation group equations we find $\alpha_{3}^{-1}\left(M_{Z}\right)=\left[\alpha_{3}^{-1}\left(M_{Z}\right)\right]_{\mathrm{MSSM}}+\Delta_{3}$, where $\left[\alpha_{3}^{-1}\left(M_{Z}\right)\right]_{\text {MSSM }}$ corresponds to the value one would have obtained within the MSSM. $\Delta_{3}$ includes corrections from additional states (below the $M_{G}$ scale) of our model:

$$
\begin{aligned}
\Delta_{3}= & \frac{3}{14 \pi} \ln \left(\frac{M_{D_{1}} M_{D_{2}}}{M_{T_{2}} M_{T_{3}}}\right)^{3} \frac{M_{3}^{8} M_{G}^{2}}{M_{T_{1}} M_{e_{H}^{c}}^{2} M_{8}^{7}}\left(\frac{M_{G}}{M_{u_{f}^{c}}}\right)^{5 n_{f}}\left(\frac{M_{G}}{M_{l_{f}}}\right)^{3 n_{f}} \\
& +\delta_{3}^{(2)} .
\end{aligned}
$$

The $\delta_{3}^{(2)}$ stands for a two-loop correction, taken into account upon numerical calculations. Between the thresholds' masses, the reasonable (and fairly natural) balance, giving successful gauge coupling unification, can be found. For instance, with the choice

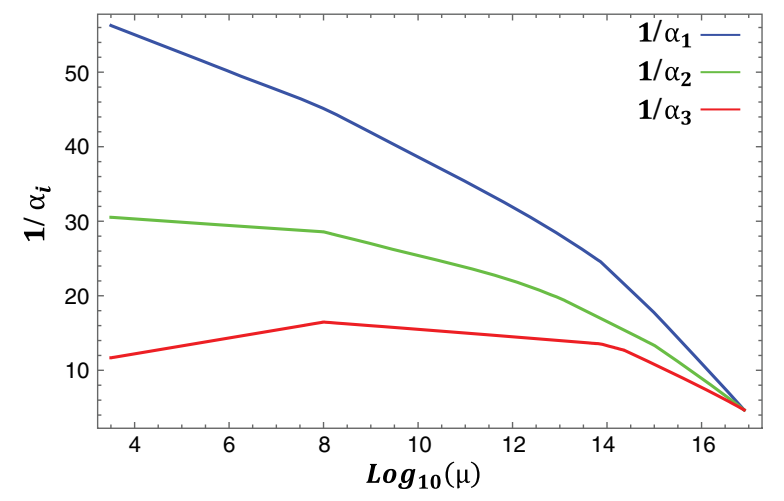

FIG. 1. Gauge coupling unification within the presented $S O(10)$ model. The spectrum of states is given in Eq. (24). 
$M_{D_{1}} \simeq 3.6 \times 10^{11}, \quad M_{D_{2}} \simeq 1.05 \times 10^{12}, \quad M_{T_{1}}=10^{8}$,

$M_{T_{2,3}} \simeq M_{G}=8 \times 10^{16}, \quad M_{8} \simeq 2.29 \times 10^{14}, \quad M_{3\left(e_{H}^{c}\right)}=10^{13}$,

$M_{u_{f}^{c}} \simeq 7.38 \times 10^{13}, \quad M_{l_{f}} \simeq 1.04 \times 10^{15}, \quad n_{f}=3$

(all masses are given in $\mathrm{GeV}$ ), the gauge couplings meet at the scale $M_{G} \simeq 8 \times 10^{16} \mathrm{GeV}$, as displayed in Fig. 1, and the unified gauge coupling is $\alpha_{G}\left(M_{G}\right) \simeq 0.214$ [22]. This shows how the presented scenario gives consistent gauge coupling unification.

\section{SUMMARY}

The main result of this paper is the novel mechanism suggested within the $S O(10)$ GUT for the Higgs particle being the light pseudo-Goldstone mode. Moreover, realizing and demonstrating the proposed mechanism, an explicit model with a fully realistic phenomenology has been constructed.

Within a scenario by the proposed mechanism, it is tempting to address in detail more phenomenological issues. Also, it would be interesting to investigate the origin of various exploited higher-order operators through renormalizable interactions, together with possibilities of suppressing the $V_{R}$ (the $45_{H}$ 's VEV in the $I_{3 R}$ direction). For this, the proposal of Ref. [10] can be applied, which as shown in Ref. [12] (within a different construction) can be very efficient when supported by anomalous or/and discrete symmetries. The latter, instead of being global, can arise from discrete gauge symmetry [23] having a stringy origin. It is tempting to use such symmetry as a flavor symmetry (gaining more motivation) and study related issues in more detail. In this paper, I preferred to stay with the minimal setup, so I have not pursued such possibilities. All these, supported by the mechanism discussed here, open wide prospect towards novel $S O(10)$ GUT model building and motivate one to study related phenomenology, such as the fermion mass pattern, neutrino oscillations, leptogenesis, and proton decay rates. The latter still needs to be probed by ongoing nucleon decay search experiments [24].

\section{APPENDIX DECOMPOSITION OF $W_{H}^{(45,16)}$}

The invariant $\left(\overline{16}_{H} 16_{H}\right)_{210} \cdot\left(45_{H}^{2}\right)_{210}$, which we assume to appear in the superpotential coupling $W_{H}^{(45,16)}$ of Eq. (4), in explicit $S O(10)$ index contractions, is

$$
\begin{gathered}
\left.\left(\overline{16}_{H} 16_{H}\right)_{210} \cdot\left(45_{H}^{2}\right)_{210}=\left\langle\overline{16}_{H}^{T}\left|B \Gamma_{i} \Gamma_{j} \Gamma_{m} \Gamma_{n}\right| 16_{H}\right\rangle \Phi^{[i j m n}\right) \\
\text { with } \quad \Phi^{[i j m n]}=45_{H}^{i j} 45_{H}^{m n}-45_{H}^{m j} 45_{H}^{\text {in }}+45_{H}^{i m} 45_{H}^{j n}
\end{gathered}
$$

Here $i, j, m, n=1-10$ and $\Gamma$ 's are gamma matrices in $S O(10)$ 's spinorial representation and $B$ is the analog of the charge conjugation operator for $S O(10)$.

With $S O(10) \rightarrow S U(4)_{c} \times S U(2)_{L} \times S U(2)_{R}$ decomposition of (2) and

$$
\begin{aligned}
& 16_{H}=F_{H}(4,2,1)+F_{H}^{c}(\overline{4}, 1,2), \\
& \overline{16}_{H}=\bar{F}_{H}(\overline{4}, 2,1)+\bar{F}_{H}^{c}(4,1,2),
\end{aligned}
$$

the relevant couplings, extracted from the operator of $W_{H}^{(45,16)}$ in terms of $S U(4)_{c} \times S U(2)_{L} \times S U(2)_{R}$ 's fragments, will be

$$
\begin{aligned}
& \frac{i}{4 !}\left(\overline{16}_{H} 16_{H}\right)_{210} \cdot\left(45_{H}^{2}\right)_{210} \rightarrow \frac{1}{2}\left(\bar{F}_{H} \Sigma^{2} F_{H}+\bar{F}_{H}^{c} \Sigma^{2} F_{H}^{c}\right) \\
& \quad-2 \bar{F}_{H}^{c} \Sigma \Sigma_{R} F_{H}^{c}+2 \bar{F}_{H}^{c} \Sigma_{R}^{2} F_{H}^{c} \\
& \quad-\left(\frac{1}{8} \operatorname{tr} \Sigma^{2}+\frac{1}{2} \operatorname{tr} \Sigma_{R}^{2}\right)\left(\bar{F}_{H} F_{H}+\bar{F}_{H}^{c} F_{H}^{c}\right)
\end{aligned}
$$

Factors in (A3) correspond to the normalization of the VEVs:

$$
\begin{aligned}
\langle\Sigma\rangle & =V_{B-L} \cdot \operatorname{Diag}(1,1,1,-3), \\
\left\langle\Sigma_{R}\right\rangle & =V_{R} \cdot \operatorname{Diag}(1,-1),
\end{aligned}
$$

where $\langle\Sigma\rangle$ and $\left\langle\Sigma_{R}\right\rangle$ are given in $S U(4)_{C}$ and $S U(2)_{R}$ group spaces, respectively. From these, one can easily obtain the results presented in Eqs. (8)-(10), (12), and (13).
[1] J. C. Pati and A. Salam, Phys. Rev. Lett. 31, 661 (1973); Phys. Rev. D 10, 275 (1974); H. Georgi and S. L. Glashow, Phys. Rev. Lett. 32, 438 (1974).

[2] H. Georgi, AIP Conf. Proc. 23, 575 (1975); H. Fritzsch and P. Minkowski, Ann. Phys. (N.Y.) 93, 193 (1975).

[3] P. Minkowski, Phys. Lett. 67B, 421 (1977); M. Gell-Mann, P. Ramond, and R. Slansky, Conf. Proc. C 790927, 315 (1979); T. Yanagida, Conf. Proc. C 7902131, 95 (1979); R. N. Mohapatra and G. Senjanovic, Phys. Rev. Lett. 44, 912 (1980).
[4] M. Fukugita and T. Yanagida, Phys. Lett. B 174, 45 (1986).

[5] S. Dimopoulos, S. Raby, and F. Wilczek, Phys. Rev. D 24, 1681 (1981); W. J. Marciano and G. Senjanovic, Phys. Rev. D 25, 3092 (1982).

[6] The solution of the DT splitting problem requires some underlying mechanism for a natural understanding of the lightness of the Higgs doublet and, at the same time, adequate suppression of the color triplet mediated nucleon decays. 
[7] S. Dimopoulos and F. Wilczek, Print-81-0600 (Santa Barbara), Report No. NSF-ITP-82-07.

[8] K. S. Babu and S. M. Barr, Phys. Rev. D 48, 5354 (1993).

[9] Z. Berezhiani and Z. Tavartkiladze, Phys. Lett. B 409, 220 (1997).

[10] S. M. Barr and S. Raby, Phys. Rev. Lett. 79, 4748 (1997).

[11] K. S. Babu, J. C. Pati, and F. Wilczek, Nucl. Phys. B566, 33 (2000).

[12] K. S. Babu, J. C. Pati, and Z. Tavartkiladze, J. High Energy Phys. 06 (2010) 084.

[13] For a recent review and extensive reference list, see S. Raby, Lect. Notes Phys. 939, 1 (2017).

[14] K. Inoue, A. Kakuto, and H. Takano, Prog. Theor. Phys. 75, 664 (1986); A. A. Anselm and A. A. Johansen, Phys. Lett. B 200, 331 (1988).

[15] Z. G. Berezhiani and G. R. Dvali, Bull. Lebedev Phys. Inst. 5, 55 (1989).

[16] R. Barbieri, G. R. Dvali, and M. Moretti, Phys. Lett. B 312 , 137 (1993); R. Barbieri, G. R. Dvali, A. Strumia, Z. Berezhiani, and L. J. Hall, Nucl. Phys. B432, 49 (1994).
[17] G. R. Dvali and S. Pokorski, Phys. Rev. Lett. 78, 807 (1997).

[18] M. Dine, N. Seiberg, and E. Witten, Nucl. Phys. B289, 589 (1987); J. J. Atick, L. J. Dixon, and A. Sen, Nucl. Phys. B292, 109 (1987).

[19] This issue needs additional care, because the triplet states of (13) have masses near $\sim 10^{8} \mathrm{GeV}$.

[20] Also, there are allowed the following operators: $\left(S+45_{H}^{2}\right) S_{1}^{3} 10_{H} 10_{H}$, and $\left(S+45_{H}^{2}\right) S_{1}^{4} 16_{H} 16_{H} 10_{H}$, which do not give any relevant contribution and are safely ignored.

[21] A similar suppression of $d=5$ proton decay, in a rather different $S O(10)$ construction, was obtained in Ref. [12].

[22] Upon analysis we have taken $\alpha_{3}\left(M_{Z}\right) \simeq 0.1187$ and for simplicity assumed that all SUSY states have masses $\simeq 1 \mathrm{TeV}$.

[23] L. E. Ibanez and G. G. Ross, Phys. Lett. B 260, 291 (1991); L. E. Ibanez, Nucl. Phys. B398, 301 (1993).

[24] S. Raby et al., arXiv:0810.4551; K. S. Babu et al., arXiv:1311.5285. 